\title{
Shaping the light amplified in a multimode fiber
}

\author{
Raphael Florentin ${ }^{1}$, Vincent Kermene ${ }^{1}$, Joel Benoist ${ }^{1}$, Agnès Desfarges-Berthelemot ${ }^{1}$, Dominique Pagnoux ${ }^{1}$, \\ Alain Barthélémy ${ }^{1}$ and Jean-Pierre Huignard ${ }^{2}$
}

Propagation of light in multimode optical fibers usually gives a spatial and temporal randomization of the transmitted field similar to the propagation through scattering media. Randomization still applies when scattering or multimode propagation occurs in gain media. We demonstrate that appropriate structuration of the input beam wavefront can shape the light amplified by a rareearth-doped multimode fiber. Profiling of the wavefront was achieved by a deformable mirror in combination with an iterative optimization process. We present experimental results and simulations showing the shaping of a single sharp spot at different places in the output cross-section of an ytterbium-doped fiber amplifier. Cleaning and narrowing of the amplifier far-field pattern was realized as well. Tailoring the wavefront to shape the amplified light can also serve to improve the effective gain. The shaping approach still works under gain saturation, showing the robustness of the method. Modeling and experiments attest that the shaping is effective even with a highly multimode fiber amplifier carrying up to 127 modes.

Light: Science \& Applications (2017) 6, e16208; doi:10.1038/lsa.2016.208; published online 24 February 2017

Keywords: adaptive optics; amplification; beam shaping; complex media; multimode optical fiber

\section{INTRODUCTION}

Large-core silica multimode (MM) optical fibers were extensively studied in the 1970s in the context of optical communications and were recognized as a major contribution to science and technology through the Nobel Prize in physics awarded to C Kao in 2009. MM fibers were progressively abandoned during the 1980s, however, because of their lower data rate transmission capacity with respect to single-mode fibers. In the same period, however, contrary to the general feeling, $\mathrm{S}$ Berdagué and $\mathrm{P} \mathrm{Facq}^{1}$ showed that spatial mode multiplexing could allow a MM fiber to carry several communication channels in parallel, which can outperform the capacity of a singlemode fiber. That proposal was revisited in recent years ${ }^{2}$ and considered as a promising way to overcome the capacity crunch foreseen in the near future with the current technology and installed network. Space division multiplexing (SDM) is a hot topic and sheds new light on the potentiality of MM fibers. However, the interest in MM fiber is not limited to optical communications. It spreads to other fields including spectroscopy, for making innovative, efficient spectroscopes $^{3}$, nonlinear optics, for an extension of soliton-based frequency conversion ${ }^{4,5}$, and imaging, where it could open new routes in endoscopy ${ }^{6}$. In that third domain, a number of techniques have been recently published that demonstrated remote imaging through step index MM optical fiber. Most of them rely on the coherent control of the fiber excitation and benefit from the availability of spatial light modulators with a high number of elements. The high degree of spatial freedom in the way light is trapped and transmitted through MM waveguides causes the delivered laser light to appear as a scattered beam with a random speckle pattern. That characteristic causes MM fiber to be unappealing for imaging. However, propagation through passive MM fibers remains deterministic even in the case of strong mode coupling such as propagation through a scattering medium. Therefore, control of light propagation through MM fibers can be achieved and hence can give access to imaging. In particular, image transmission has been demonstrated with methods derived from those used for focusing through scattering media. For example, some techniques are based on the use of a transmission matrix (TM), in which the image is reconstructed after computer post-processing ${ }^{7}$ or directly at the fiber end facet ${ }^{8}$. Some others exploit digital phase conjugation ${ }^{9}$. Adaptive spatial shaping in combination with optimization routines is also efficient to shape a multimode output ${ }^{6}$. It was recently demonstrated ${ }^{10}$ that even in the case of mode coupling, a desired intensity profile can be formed at the output of a passive MM fiber thanks to an adaptive shaping of the input field wavefront with a spatial light phase modulator. Narrow beam spots were delivered at the fiber output facet and were positioned at different locations in the core section of $50 \mu \mathrm{m}$ in diameter. The system was used to image a target through the fiber by remote laser scanning of the object.

One can now ask what happens if the MM fiber or the scattering medium transmission is no longer considered as a lossless and gainless medium. One can ask which occurs if the transmission medium can no longer be represented by only a linear transformation (such as a TM). Only a few studies have investigated propagation through a scattering medium with a localized absorption area ${ }^{11,12}$, and none considered control of the output light field. They rather targeted the identification of the maximal transmission channel. Moreover, MM fibers with gain, such as MM rare-earth-doped fiber amplifiers, have

${ }^{1}$ XLIM Research Institute, Université de Limoges-Centre National de la Recherche Scientifique, UMR 6252, 87060 Limoges, France and ${ }^{2}$ Institut Langevin, ESPCI-ParisTech, 75238 Paris, France

Correspondence: A Barthélémy, Email: alain.barthelemy@xlim.fr

Received 3 March 2016; revised 22 August 2016; accepted 25 August 2016; accepted article preview online 29 August 2016 
not been extensively considered in the context of light transport control. Such a medium also loses the linear description of light transmission, which applies to the same passive non-doped fiber. Therefore, one can wonder whether it is possible to shape the output field intensity pattern of an MM fiber in the amplification regime. One first issue could be that, even in the small signal gain regime, the amplification is not uniformly shared by the guided modes (or by the transmission channel for bulk scattering media). In the context of SDM optical communications, this requires gain equalization methods to be found ${ }^{13,14}$. In addition, linearity of the light-field transmission is generally lost in an amplifying MM fiber with the onset of gain saturation. The multimode field creates in the waveguide a threedimensional (3D) complex interference intensity pattern and forms a $3 \mathrm{D}$-speckled structure so that depletion of the gain at high signal intensity is uniform neither in the fiber cross section nor along the axis. Gain competition in the modal population is one of the possible consequences of such non-uniform saturation. Therefore, this suggests that the TM approach is inappropriate, and one could wonder whether the other beam shaping processes previously applied to MM fibers and scattering samples can work in the amplification regime. In this paper, we investigated, for the first time to our best knowledge, the ability of the adaptive wavefront shaping technique to tailor the output pattern of an MM fiber amplifier. We show by simulation and experiments that the adaptive technique is able to change the amplified light speckle into a desired profile, such as a single focus, several spots or a pure mode, directly at the output of the fiber. The iterative optimization process is also able to cope with the nonlinear behavior of the MM fiber with respect to its light transport properties in the regime of gain saturation. The fiber we have used in most of the experiments is not a standard step index MM fiber but a multimode multicore (MM-MC) fiber with ytterbium ( $\mathrm{Yb})$ doping. The fiber has an inner guiding section of nearly $50 \mu \mathrm{m}$ in diameter and guides 24 linearly polarized (LP) supermodes (two polarizations). Bright spot formation at the output of a single-core step index MM amplifying fiber carrying as many as 254 LP modes (two polarizations) has also been numerically and experimentally demonstrated.

\section{MATERIALS AND METHODS}

\section{Experimental setup}

The experimental setup is depicted in Figure 1. It starts with an amplified laser diode at $1064 \mathrm{~nm}$ for the coherent light source. The laser diode is coupled with a polarization-maintaining fiber so that a perfectly single-mode LP beam of a nearly Gaussian profile is provided at the output. A telescope expands the beam before it illuminates a deformable mirror (DM) of a square surface (Boston Micromachines Multi-DM, Cambridge, MA, USA). The laser wave overfills the aperture of the DM, which reflects the beam's central part only. The coherent field shaped by the deformable continuous surface of the DM $(12 \times 12$ actuators) is precisely imaged with reduction onto the input face of the optical fiber by a second telescope. The MM-MC optical fiber is made of an array of $19 \mathrm{Yb}$-doped cores of $7 \mu \mathrm{m}$ in diameter (0.065 numerical aperture (NA)) in a triangular lattice of $10.5 \mu \mathrm{m}$ pitch covering a hexagonal section of $\sim 50 \mu \mathrm{m}$ in diameter. The distance between the cores and the refractive index difference $\left(1.45 \times 10^{-3}\right)$ are such that the cores are strongly coupled. Thus, the fiber behaves like an MM waveguide whose supermodes have been computed using a finite element method mode solver. The calculations show 12 propagation modes and therefore 24 modes if we count the two polarization orientations. The central part of the structure is surrounded by a first cladding with an octagonal shape of $\sim 480 \mu \mathrm{m}$ in diameter to guide the pump beam $(\mathrm{NA}=0.46)$ and then by a second cladding. The fiber input face was oriented such that one axis of the core lattice was in line with the one of the square arrays of actuators of the DM. The fiber was $1.5 \mathrm{~m}$ long and coiled with a loop of $35 \mathrm{~cm}$ in diameter between its two holders. On the output side, pump light from the laser diode was injected in free space into the MM fiber by a dichroic beam splitter and imaging optics. Its power varied in the range of $0-50 \mathrm{~W}$. A hard aperture located in the nearfield image plane served to filter out spurious signal light exiting from the cladding. The final part of the setup consisted of a power meter and cameras to obtain real-time near-field and far-field patterns. They are connected to a laptop for computation and driving of the DM surface.

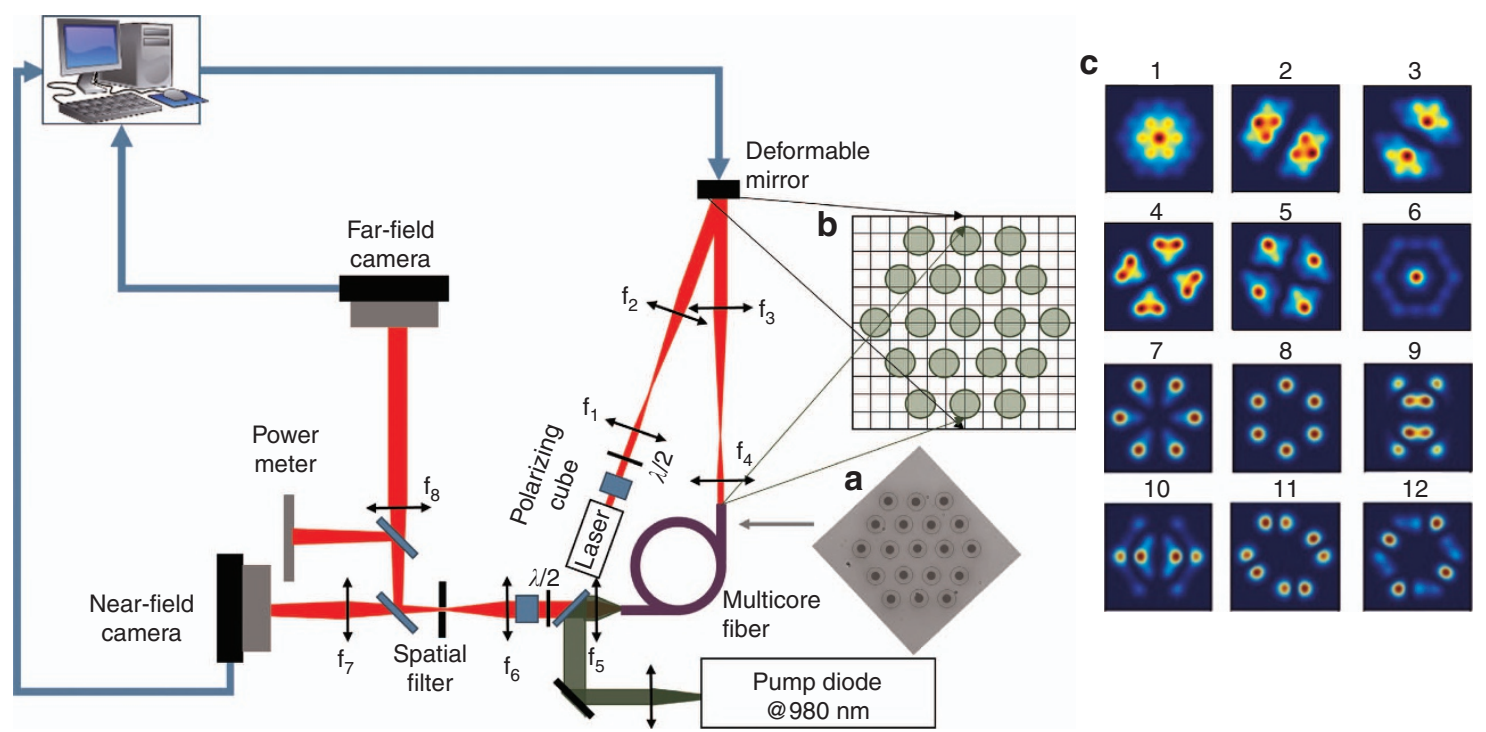

Figure 1 Schematic of the experimental setup including (a) an image of the MM-MC Yb-fiber (in gray), (b) a drawing of the downscale imaging of the DM surface on the fiber input face and (c) the 12 supermode patterns. 


\section{Beam shaping procedure}

The DM being set with a flat surface, we first optimized the coupling of the input signal into the MM-MC fiber. It was impossible in that situation to obtain an output pure mode pattern and particularly to have the fundamental mode only. In all cases, the observed fiber output corresponded to the superposition of several modes and appeared as a speckled laser beam because of mode coupling. To shape the output beam profile, it is proposed to structure the input field wavefront. The transverse distribution of the phase in the launched beam governs its modal expansion. After propagation, the excited modes reach the fiber exit with a change in their phase relationships because of their different propagation constants. Their coherent summation generates the light-field distribution appearing on the fiber output face. For determining the input phase pattern required to obtain a desired output, the more complete approach consists of the prior measurement of the transmission matrix (TM) of the system as it is performed with scattering media. That requires a long characterization step, but, provided that the optical fiber is maintained in the same working condition, the knowledge of the TM directly gives the shape of the DM surface necessary to obtain any requested output. This is valid even in the presence of mode couplings, but the fiber must behave as a linear system. This is unlikely to be the case for a multimode fiber amplifier because of inhomogeneous gain among the modes and because of saturation and gain competition. In addition, changing the pump power should modify the thermal equilibrium of the fiber so that the TM should be measured again after any change in the operating conditions. Another option to obtain a desired amplified beam figure consists of an adaptive shaping of the transverse phase through an optimization process. It was shown recently with a passive MM fiber that it is possible to obtain a desired intensity pattern at the output through that process ${ }^{10}$. This is a much simpler approach, but to be fast and efficient, the approach requires a proper choice of the parameter to optimize and a proper choice of the optimization method.

We have used different parameters, also named objective functions (OF) in the frame of optimization, which seem to be suited to an MM fiber with gain:

$$
\begin{aligned}
\mathrm{OF}_{1}= & \iint_{A}\left|\psi_{\text {out }}(x, y)\right|^{2} \mathrm{~d} x \mathrm{~d} y / \iint_{R}\left|\psi_{\text {out }}(x, y)\right|^{2} \mathrm{~d} x \mathrm{~d} y \\
\mathrm{OF}_{2}= & \left(\iint_{R} \psi_{\text {out }}(x, y) \psi_{\text {des }}^{*}(x, y) \mathrm{d} x \mathrm{~d} y\right)^{2} / \\
& \left(\iint_{R}\left|\psi_{\text {out }}(x, y)\right|^{2} \mathrm{~d} x \mathrm{~d} y \iint_{R}\left|\psi_{\text {des }}(x, y)\right|^{2} \mathrm{~d} x \mathrm{~d} y\right)
\end{aligned}
$$

where $\psi_{\text {out }}(x, y)$ denotes the current output field pattern, and $\psi_{\text {des }}$ $(x, y)$ denotes the desired mode, $A$ being the area covered by the desired profile and $R$ being the fiber core complete cross-section. Most of the experiments reported below were obtained with the use of $\mathrm{OF}_{1}$, which is a well-appropriate criterion to shape a single (or a few) light spot(s). $\mathrm{OF}_{2}$ was used for optimization and for selection of a pure specific mode. Regarding the optimization method we have chosen, it is derived from phase shifting interferometry ${ }^{15}$. For each spatial sample (pixel) of the input beam on the DM, three measurements of the objective function are necessary to compute the command applied to the spatial phase modulator. One is made with the modulator at rest, and the other two are recorded for a phase modulation of $+/-\phi_{\mathrm{opt}}$, giving three values of the measured parameter OF denoted by $S_{0}, S_{+}$and $S_{-}$. The optimal phase is then computed from the expression ${ }^{15}$

$$
\phi_{\mathrm{opt}}=\tan ^{-1}\left(\frac{\left(S_{+}-S_{-}\right)(1-\cos (\delta))}{\left(2 S_{0}-S_{+}-S_{-}\right) \sin (\delta)}\right)
$$

and then applied to the DM before considering the next pixel. One pass over all mirror elements is sufficient to obtain the optimal wavefront.

\section{Modeling}

We developed a numerical tool that models the operation of the whole setup. In a preliminary step, the eigenmode fields (supermodes) and propagation constants have been computed. Computation of their respective small-signal gain was achieved considering the overlap between the guided mode intensity and the rare-earth-doped area. The latter was discrete and limited to the 19 high index zones of the lattice. The modeling then proceeds as follows. We assumed that the laser beam had an initial plane wavefront and almost uniformly covered the DM, whose surface was discretized in $12 \times 12$ elements. The wavefront shaped by the DM was imaged onto the MM-MC fiber and expanded on its eigenmode basis. The modes were propagated on the MM-MC fiber length divided in a few segments. We introduced mode coupling at the transition between two segments through the addition of a linear transverse phase gradients with various slopes and orientations, which mimic sharp bending. The effective gain for each mode, including the saturation effect, was calculated at the input of each segment considering the mode intensity. The transverse profile of the output beam was built up by coherent summation of the transmitted modes and served to derive the value of the OF. That parameter was used to carry on the optimization process and to obtain after iteration the final settings of the DM. In the last step, we computed the final intensity pattern at the fiber amplifier output.

\section{RESULTS AND DISCUSSIONS}

\section{Adaptive shaping of an MM-MC fiber amplifier output beam}

In a preliminary step, with the pump laser switched off, we have chosen to shape the signal input wavefront to obtain a beam confined in a single narrow spot on the fiber output facet. Therefore, the phase profile was set to maximize the output power detected on a circular area of $5 \mu \mathrm{m}$ in diameter, as measured by a camera on an image of the fiber exit face. The location and size of the target were chosen to approximately match the center core of the MM-MC fiber. Figure $2 \mathrm{a}$ shows the output intensity pattern for a flat DM surface, and Figure $2 b$ shows the one recorded after optimization of the wavefront shaping. The hexagon in the dashed white line represents the overall core section, and the small dashed circle denotes the target. The images clearly attest of the focusing of the output beam on a sharp, bright spot with a diameter of $7.5 \mu \mathrm{m}$ (full width-half maximum) corresponding to $\sim 1 / 7$ th of the large hexagonal core. Only a weak fraction of the output beam power remained visible in the close neighborhood of the spot. It was also possible to locate the spot on various positions in the core section-for instance, close to the boundary of the cladding. The result was repeatable and stable in our laboratory environment, so once the DM was adjusted and kept fixed, the desired output beam was preserved for at least $1 \mathrm{~h}$ without updating the mirror command. We then switched on the laser diode at $980 \mathrm{~nm}$, and the pump power was raised until we obtained a maximum amplification of $17 \mathrm{~dB}$. We repeated the previous optimization procedure. The DM being in a flat position, the output pattern changed with respect to Figure 2a because of 

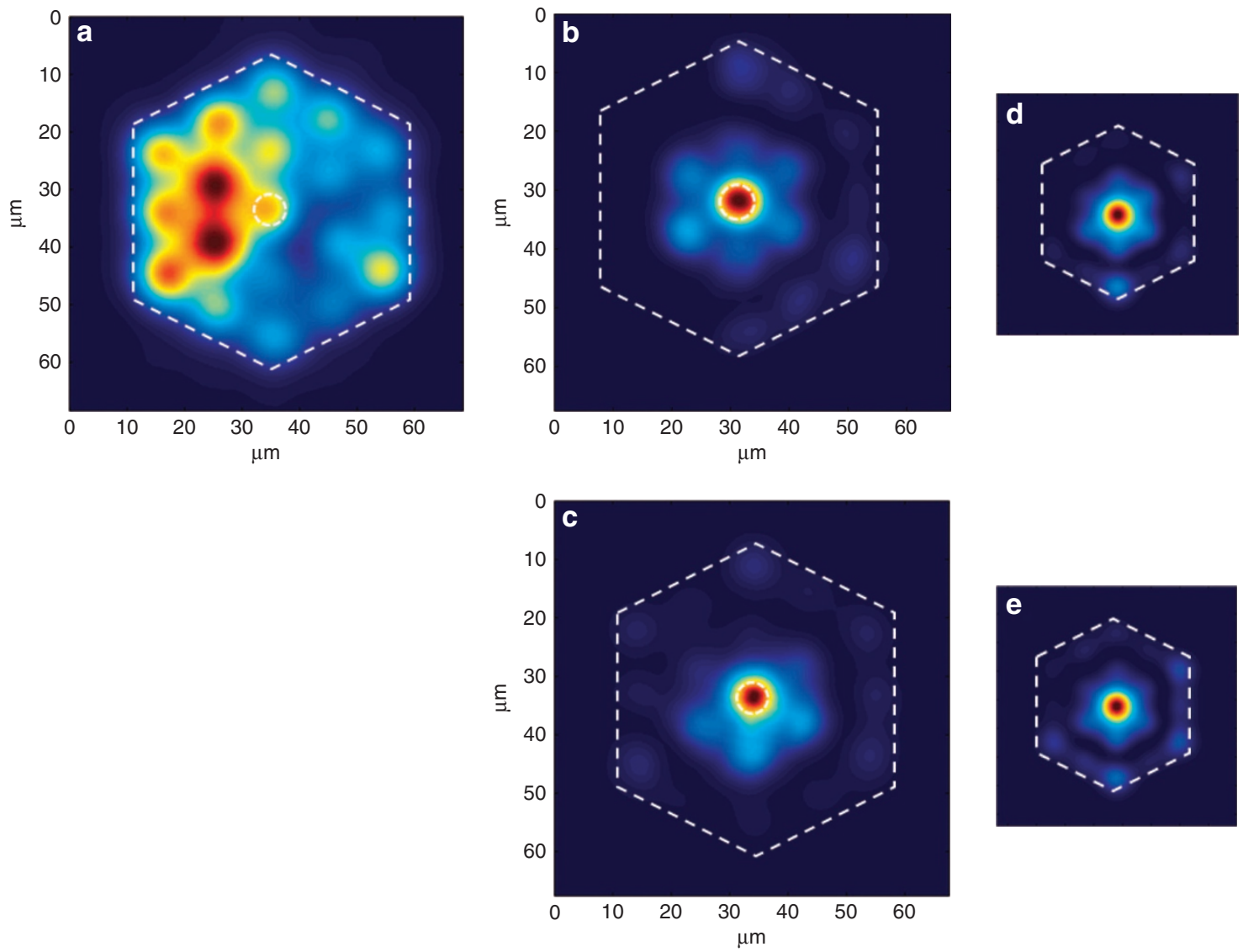

Figure 2 Output patterns recorded on the exit face of the MM-MC Yb-doped optical fiber: (a) without wavefront profiling, (b) after wavefront shaping for focusing on the central core without gain and (c) after wavefront shaping with 17-dB small signal gain. Insets show numerical results of the modeling of the whole system for the passive (d) and active (e) regimes, in good agreement with the observations.

thermal effects and because of mode-dependent gain. Computations indicated that the amplification varies by almost $6 \mathrm{~dB}$ among the modes, the higher-order modes exhibiting the highest gain and the fundamental mode the lowest. One run of the adaptive shaping, maintaining the same targeted output as previously, led to the image shown on Figure 2c. Focusing the output pattern on the central core at the fiber exit face was effective both without gain (b) and with gain (c) in agreement with the modeling (Figure $2 \mathrm{~d}$ and 2e). Almost no change in coupling efficiency was observed at the input fiber side. The focusing efficiency was even improved by nearly $30 \%$. The capability to concentrate the light on a single bright spot was therefore preserved in the amplification regime $(\sim 17 \mathrm{~dB})$. The dynamics of the optimization process for the shaping remained smooth as in the passive case. The evolution of the OF in the course of the DM setting was similar; that is, the measured parameter continuously increased, whereas the system adjusted the imposed phase pixel by pixel. This was expected because the algorithm is of the coordinate ascent type. Optimization of the mirror structure configuration here takes $\sim 120 \mathrm{~s}$, a duration that is fixed by the slow interface between the camera and the LabVIEW software on the desktop. In an improved setup, a simple photodiode performed the same work within a $50 \mathrm{~ms}$ time lapse. We checked that the system gave the same output pattern and phase chart after optimization when starting from various initial conditions. Once an optimization has led to the DM settings corresponding to a given spot, they can be recorded. A few hours later, the same output spot could be recovered simply by applying the recorded DM command.

It is worth mentioning that the wavefront shaping at the input to obtain the desired target spot at the output was different with and without gain because of the impact of pumping inhomogeneity because of differential effects of the thermal load and because of the non-uniform gain among the modal population. As evidence, we report in Figure 3 the phase map introduced by the deformable mirror on the input laser beam after an experimental optimization of the profiling for the passive MM-MC fiber (Figure 3a) and for the amplifying fiber at $17-\mathrm{dB}$ gain (Figure $3 \mathrm{~b}$ ). There was a clear difference in the two optimal wavefronts, demonstrating that the shaping made for the passive operation is unsuited for the amplification regime. The same conclusion was derived from the simple observation of the near field starting from the situation where the output beam formed a single spot with the unpumped fiber (Figure 4a). Maintaining a fixed DM setting, the output figure turned quickly to a speckle covering a larger fraction of the guiding structure when we switched on the pump laser and raised the power (Figure 4b). Numerical modeling of the whole system indicated that the mode expansion of the input field required to obtain the desired output spot evolved with the onset of gain. We emphasize also the fact that, although the amplified beam appeared at the exit as a sharp spot, the guided field covered the entire cross section $\sim 1 \mathrm{~mm}$ back from the output face and throughout the fiber. This means that the choice of a narrow output field does not favor the 
a

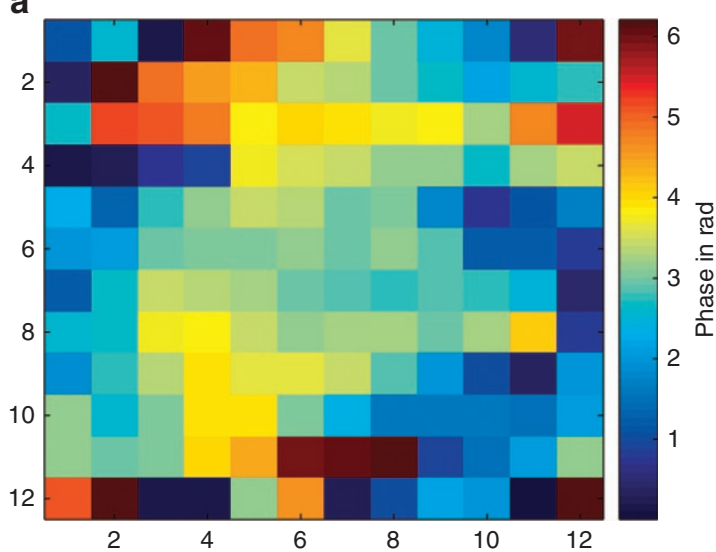

b

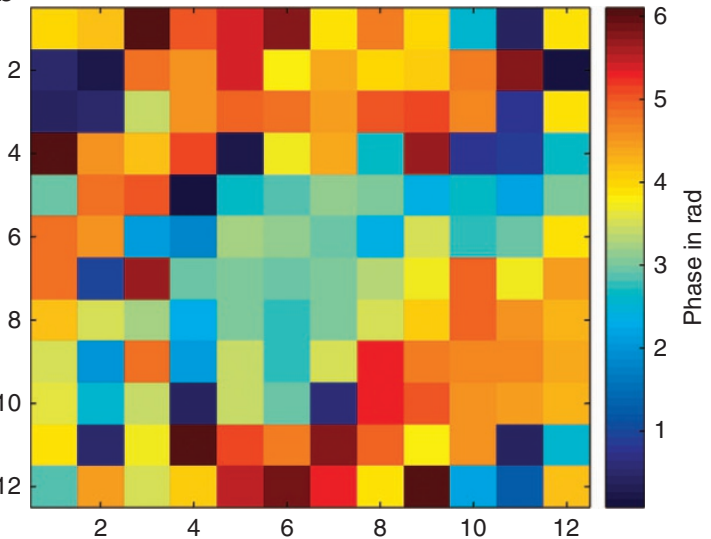

Figure 3 Experimental phase pattern imprinted on the Gaussian laser beam at the input to obtain a single bright spot on the MM fiber output without (a) and with (b) gain (unsaturated).
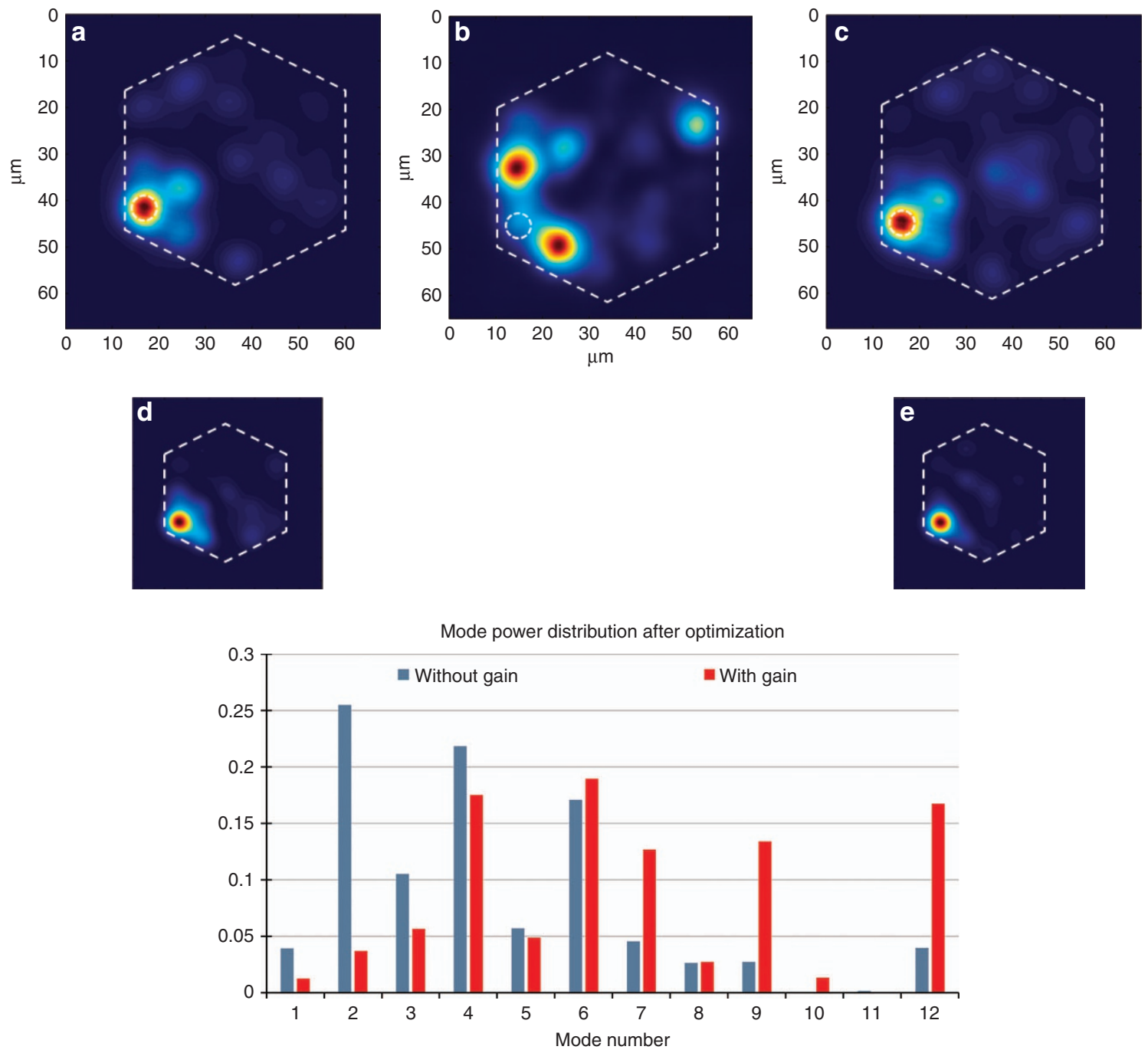

Figure 4 Concentration of the output power in a bright spot on the outer ring of the multicore fiber. Typical recordings of the MM fiber output pattern (a) in the passive regime; (b) in the amplification regime maintaining the same input wavefront as in the passive case, in which the shaping is lost; and (c) in the amplification regime after a new round of adaptive shaping, in which the focus is restored. The experimental results are in agreement with the numerical results (d) and (e) given in the two insets. With amplification, the mode expansion required to obtain the desired pattern evolves significantly with the highorder modes carrying more power, as the modeling shows (bottom). 
onset of nonlinear effects because the guided radiation effective area, averaged over the propagation distance,

$$
\left\langle A_{\mathrm{eff}}\right\rangle=\frac{1}{L} \int_{0}^{L} \frac{\left(\iint I(x, y) \mathrm{d} x \mathrm{~d} y\right)^{2}}{\iint I(x, y)^{2} \mathrm{~d} x \mathrm{~d} y} \mathrm{~d} z
$$

is not significantly modified by the shaping. It was also possible to tune the position of the amplified spot on any of the 19 cores of the Yb-doped multiple core fiber amplifier similarly to what can be accomplished in a passive multimode waveguide. A typical example is reported in Figure 4, where again one can compare the case of an optimized shaping without gain (Figure 4a) with one of a shaping achieved with a $17-\mathrm{dB}$ small signal gain (Figure 4c). As in the previous linear situation (no gain), the shaped output remained extremely stable without any alteration of the mirror settings after the initial optimization step. No permanent servo-control was required. The next question could be whether the adaptive shaping is robust with respect to gain saturation. Saturation of the gain should add more complexity in the operation of the amplifier, leading to stronger nonlinear behavior. We investigated that regime both in experiments and through modeling considering an input signal whose power level was increased by one order of magnitude. The gain saturation did not modify the previous observations. By raising the input signal level, we obtained a drop in the gain (source limited) by more than $-6 \mathrm{~dB}$ while maintaining efficient control of the beam cross-section and good concentration of the output power on a single spot. As a typical example, Figure 5 presents two images of the amplifier output intensity distribution after shaping to obtain a bright central spot. One was recorded with low signal input power (Figure 5a) in the regime of small signal amplification, and the other was recorded with stronger signal power in the saturation regime (Figure $5 b$ ). The pump power was held fixed. The two figures are almost identical and attest that saturation does not prevent the shaping capability of the investigated system.

\section{MM-MC fiber amplifier adaptive far-field shaping}

Profiling of the amplified beam was also achieved in the far field. The experimental setup was modified to image the fiber output diffraction pattern onto the camera used for the optimization procedure.
The objective function was the power collected in a circular area whose diameter was chosen to be significantly smaller $(21 \mathrm{mrad})$ than the one corresponding to the fiber NA (130 mrad full aperture). Thus, the input wavefront shaping served here to shrink the output beam far-field pattern and bring it close to that of the fundamental supermode of the fiber amplifier. The performance of the device in this new working mode is illustrated in Figure 6. The left hand side image corresponds to the initial diffraction pattern of the MM amplifier output. The circle in the dashed white line gives the location and surface fixed for the measurement of the OF. After shaping of the input signal, we obtained at the amplifier exit the far-field pattern reproduced on the right hand side of Figure 6. The far-field pattern that initially covered a 93-mrad wide area was confined in a narrow lobe of 27 -mrad width (full width at $1 / e^{2}$ in intensity). The peak intensity in the encircled area was 18 times higher with respect to the initial starting state. These data, together with complementary experimental observations and modeling, demonstrate that the input wavefront profiling may be exploited to obtain a desired beam shape also in the far field. One could conclude from Figure $6 \mathrm{~b}$ that structuring of the laser beam has simply led to the excitation of the fundamental mode of the fiber. The near-field intensity image of the amplified output recorded for the situation of Figure $6 \mathrm{~b}$ proves that this was not the case. As shown in Figure 7, the optimization did not result in the fundamental mode selection when a low divergence was sought. Coupling in the fiber and mode competition in the amplifier may explain that observation. It was also possible to steer the amplified beam direction by moving the target area on the far field image in the optimization process as was achieved in the near field.

\section{Gain with beam shape control}

We have studied the impact of beam shaping at the amplifier output on the effective gain. Measurements of the power ratio at the fiber output with and without pumping gave straightforward access to the amplification level. At first, we investigated the case of beam shaping in the near field. Comparison of the gain values measured with plane wave excitation and with a shaped input wavefront resulted in different behavior owing to the position of the desired spot. For a central position, a slight drop in the power gain was observed under beam control. We mean that by comparison with a standard Gaussian beam excitation for which the fiber delivered a multimode amplified
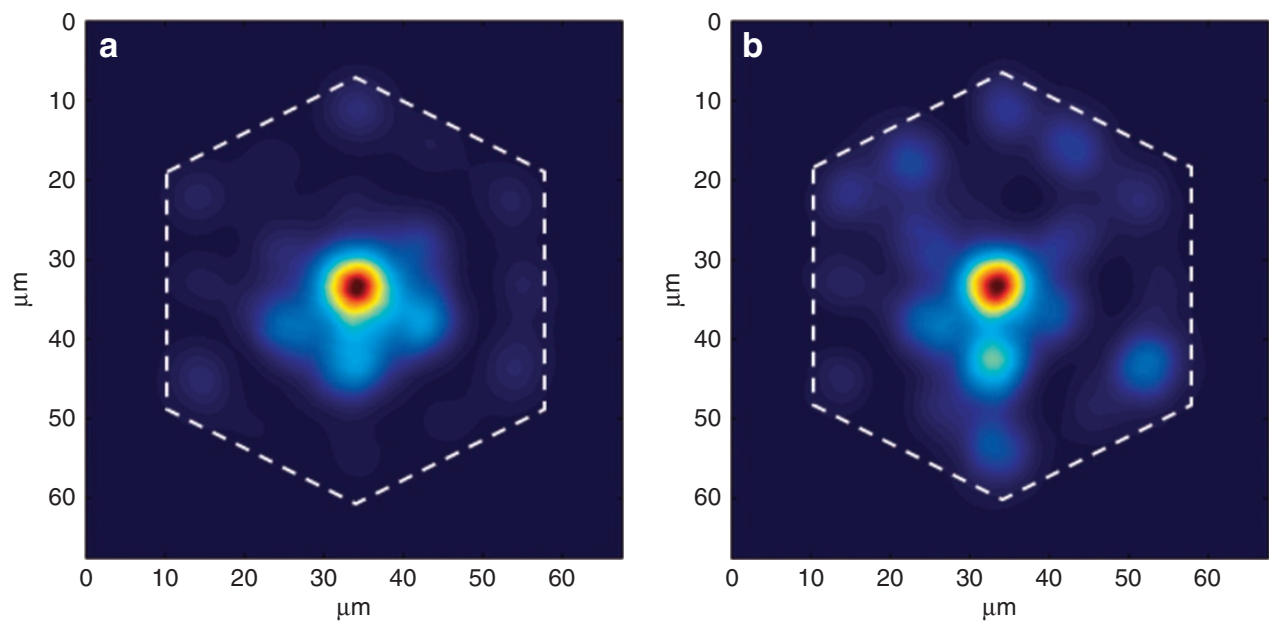

Figure 5 Comparison of the pattern recorded at the amplifier output for (a) the small signal amplification regime and for (b) amplification in the saturation regime ( $-6 \mathrm{~dB}$ gain drop) with concentration of the amplified light on a central spot. 

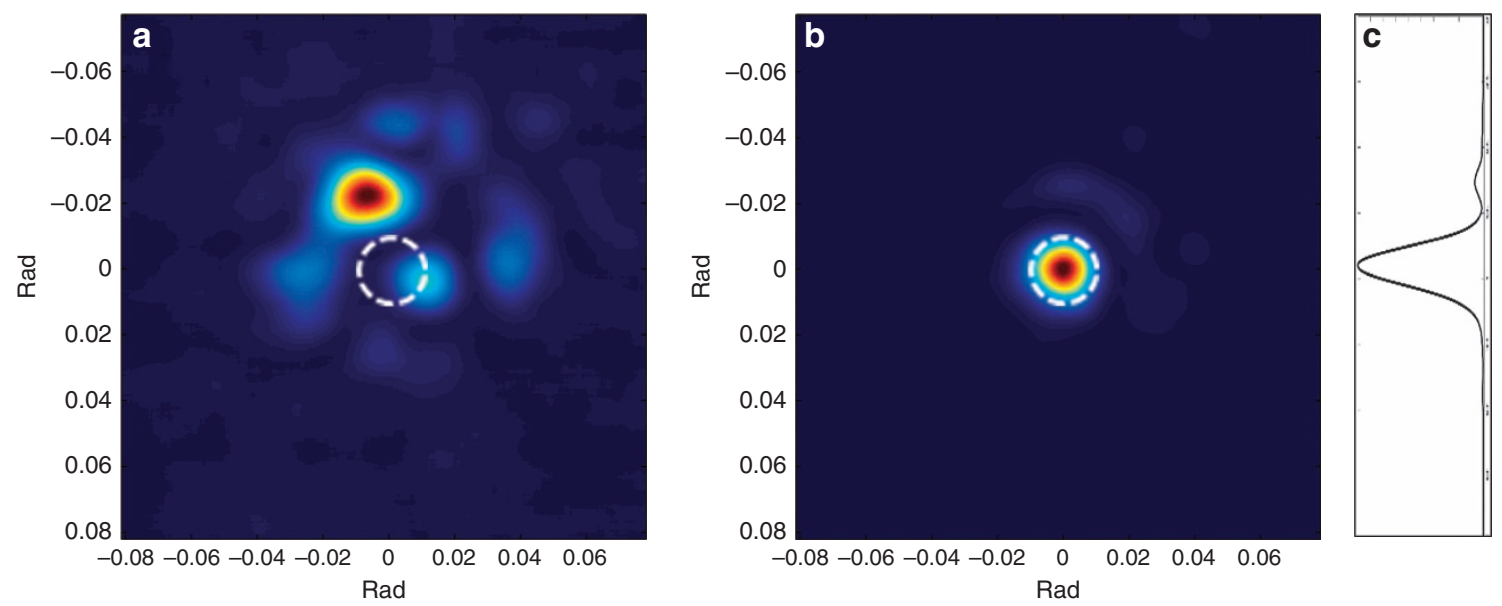

Figure 6 Experimental far-field pattern of the multicore fiber amplifier before wavefront shaping (a), final pattern after optimization of the DM surface to obtain, in the desired orientation (dashed white circle), a single narrow diffraction lobe (b) and cross-sectional profile (c).

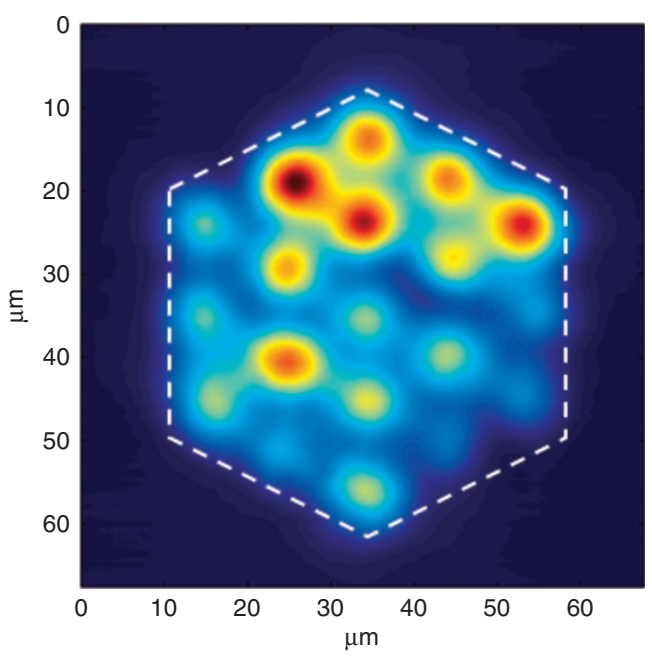

Figure 7 Near-field intensity at the MM fiber amplifier output recorded after optimization in the far field corresponding to Figure $6 \mathrm{~b}$. Clearly, the pattern does not correspond to the fundamental mode profile of the structure.

wave, the profiled wavefront excitation gave an amplified beam confined in the central core of the fiber at the expense of $24 \%$ gain reduction. In contrast, a significant gain improvement was obtained for a desired spot fixed on the outer ring of the MM-MC fiber core. In that situation, we measured a gain twice the value of that corresponding to a standard beam input and associated with a multimode speckled output. That can be explained by the fact that high-order modes exhibit a stronger gain than low-order modes. The modeling indicated that to obtain a laser spot located at the periphery of the amplifier cross-section, the shaped input beam couples more into the high-order modes by comparison with a plane wave excitation. This might explain the gain improvement in that case. The same behavior was observed in the case of saturation, although the gain reduction for a center spot became negligible $(-10 \%)$.

Concerning beam shaping in the far field, the conclusion of our experiments is slightly different. The gain changed by $-7 \%$ only when we applied the mirror deformation to reduce the amplified beam divergence and fix its direction on the axis. A similar evolution was observed above the saturation threshold.

Therefore, the price paid for tailoring the amplifier output beam is generally weak, and in some cases, beam shaping improved the effective gain by as much as $+3 \mathrm{~dB}$.

\section{Extension to a highly multimode fiber amplifier}

The modeling tool we have developed has been used to investigate whether the shaping capability, demonstrated above with an Yb-doped fiber carrying 12 modes on a linear polarization state, can be extended to a multimode amplifier with a larger number of modes. We thus considered a step index MM fiber with ten times more guided modes (127 LP modes on one polarization). The $\mathrm{Yb}$ doping was assumed to be uniform in the fiber core cross-section. Based on a commercialized deformable mirror from Boston Micromachines (Kilo-DM-CS), the wavefront shaping was realized on a circular surface with 952 pixels. The simulations demonstrated that a bright single spot of light could be obtained at the output of the MM fiber amplifier after optimized structuration of the input phase pattern (see Supplementary Information). Finally, we obtained an $\mathrm{Yb}$-doped $\mathrm{MM}$ fiber sample whose characteristics corresponded to those of the modeling. It was pumped at $980 \mathrm{~nm}$ by a laser diode providing up to a 21-dB gain with a 1.3-m long fiber piece. Shaping of the amplified output in a single spot of light was obtained in various places on the fiber core as with the previous multicore fiber (see Supplementary Information). Some examples of experimental results are reported in Figure 8 for the passive gainless case (Figure 8a) for the changes further induced by the addition of gain (Figure $8 \mathrm{~b}$ ) and for the restoration of the shaping with gain thanks to a new optimization (Figure 8c).

\section{CONCLUSION}

We investigated the relevance for multimode amplifying fibers of a recent technique for obtaining a desired output beam at the exit of a MM optical fiber. It is based on an adaptive patterning of the input signal beam by means of a deformable mirror associated with an optimization process. The method does not require a reference beam. The experiments were carried out first with an Yb-doped double cladding MM fiber of $\sim 50 \mu \mathrm{m}$ in core diameter, which was made of an array of 19 coupled waveguides. It was demonstrated for the first time to our knowledge that the adaptive wavefront shaping approach 

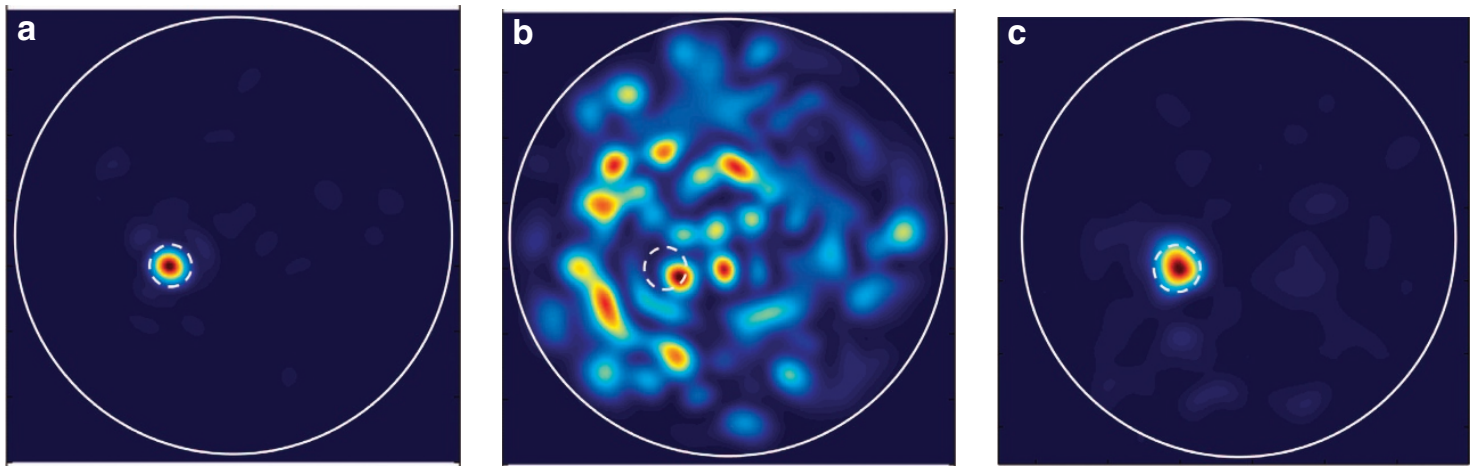

Figure 8 Highly multimode fiber amplifier, experimental output patterns (a) after adaptive structuring of the input wavefront to obtain maximum power in the small target area bounded by the white dotted line without gain; (b) after switching on the gain, while maintaining the mirror profile with the passive case setting; and (c) after a restart of the adaptive profiling for shaping with a gain of $\sim 20 \mathrm{~dB}$.

still works for the control of the near-field pattern at the exit of a strongly multimode amplifying medium. Experiments and numerical simulations support that conclusion. The amplified output field, resulting from the coherent superposition of the transmitted modes, was chosen to take the form of a single narrow light spot covering only a fraction of the multimode core. By suitable tailoring of the input wavefront, it has been moved in various positions in the core cross section. The shaping capability of the technique was preserved despite the mode coupling, the inhomogeneous gain among the guided modes and the gain competition occurring in the saturation regime. Transition between the linear and nonlinear transmission regimes according to gain saturation was investigated (see Supplementary Information). Profiling of the amplified beam in the far field was also demonstrated. By comparison with the standard excitation of the MM amplifier (wide Gaussian beam, nearly plane wavefront), the output beam adaptive control only weakly changed the effective gain in the more general situation. However, for some specific choice of the amplified spot position that favored the excitation of high-order modes, it was possible to significantly improve the gain $(+3 \mathrm{~dB})$. In most of the experiments, we have used a camera for detection, and the objective function was computed from the image data. Finally, a single photodiode was used for detection providing a speed increase by orders of magnitude for the adaptive shaping. The size of the sensor and its location in the fiber face image were adapted to obtain the expected spatial filtering. In a second step, we used a short piece of MM Yb-doped fiber with approximately ten times more guided modes. Amplified light shaping was also achieved with this fiber with even better performance. The experiments reported in the paper were carried out at a power level of approximately $1 \mathrm{~W}$. Because of the wavefront structuration giving rise to the output pattern, control was performed on the input side of the fiber amplifier by a deformable mirror, which should be compatible with high-power amplification. This could offer a new way to reach high-power amplification while preserving high beam quality. A standard MM rare-earth-doped fiber is easy to fabricate and cheap. By focusing the output energy of such an MM amplifier on one spot, adaptive input wavefront shaping restores a high-brightness beam without sacrificing the gain. For scanning of high-power radiation, the system can also move the laser spot into a different position or prepare a multi-spot pattern that could be relevant for some applications. The approach is compatible with MM fiber amplifiers of larger cross section and with MM fiber amplifiers which support a higher number of modes than the one used in the last experiments. Amplification of short pulses in a multimode amplifier could also be considered because of the underlying connection between space and time and between mode pattern and mode group delay. In particular, it was shown that adaptive spatial control allows mitigation of group delay dispersion in a multimode waveguide ${ }^{16,17}$. There is also a close analogy with scattering media in which input wavefront shaping has led simultaneously to the focusing of light intensity and to the compression of ultrashort pulses ${ }^{18}$.

\section{CONFLICT OF INTEREST}

The authors declare no conflict of interest.

\section{ACKNOWLEDGEMENTS}

The authors acknowledge funding from the French Agence Nationale de la Recherche in the frame of the POMAD project (14-CE26-0035-01). We thank Dr Marwan Abdou Ahmed from IFSW, Universitat Stuttgart, Germany, for providing the MM-MC fiber and Thales Research and Technology for providing the $\mathrm{MM} \mathrm{Yb-doped} \mathrm{fiber.}$

1 Berdagué S, Facq P. Mode division multiplexing in optical fibers. App Opt 1982; 21: 1950-1955.

2 Richardson DJ, Fini JM, Nelson LE. Space-division multiplexing in optical fibres. Nat Photon 2013; 7: 354-362.

3 Redding $\mathrm{B}, \mathrm{Cao} \mathrm{H}$. Using a multimode fiber as a high-resolution, low-loss spectrometer. Opt Lett 2012; 37: 3384-3386.

4 Wright LG, Christoulides DN, Wise FW. Controllable spatiotemporal nonlinear effects in multimode fibres. Nat Photon 2015; 9: 306-310.

5 Wright LG, Wabnitz S, Christodoulides DN, Wise FW. Ultrabroadband dispersive radiation by spatiotemporal oscillation of multimode waves. Phys Rev Lett 2015; 115: 223902.

6 Di Leonardo R, Bianchi S. Hologram transmission through multi-mode optical fibers. Opt Express 2011; 19: 247-254.

7 Choi Y, Yoon C, Kim M, Yang TD, Fang-Yen C et al. Scanner-free and wide-field endoscopic imaging by using a single multimode optical fiber. Phys Rev Lett 2012; 109: 203901.

8 Čižmár T, Dholakia K. Exploiting multimode waveguides for pure fibre-based imaging. Nat Commun 2012; 3: 1027.

9 Papadopoulos IN, Farahi S, Moser C, Psaltis D. Focusing and scanning light through a multimode optical fiber using digital phase conjugation. Opt Express 2012; 20: 10583-10590.

10 Mahalati RN, Askarov D, Wilde JP, Kahn JM. Adaptive control of input field to achieve desired output intensity profile in multimode fiber with random mode coupling. Opt Express 2012; 20: 14321-14337.

11 Liew SF, Popoff SM, Mosk AP, Vos WL, Cao H. Transmission channels for light in absorbing random media: from diffusive to ballistic-like transport. Phys Rev B 2014; 89: 224202.

12 Hao X, Martin-Rouault L, Cui M. A self-adaptive method for creating high efficiency communication channels through random scattering media. Sci Rep 2014; 4: 5874.

13 Jung Y, Kang QY, Sahu JK, Corbett B, O'Callagham J et al. Reconfigurable modal gain control of a few-mode EDFA supporting six spatial modes. IEEE Photon Technol Lett 2014: 26: 1100-1103. 
14 Mahalati RN, Askarov D, Kahn JM. Adaptive modal gain equalization techniques in multi-mode Erbium-doped fiber amplifiers. J Lightwave Technol 2014; 32: 2133-2143.

15 Huang PS, Zhang S. Fast three-step phase-shifting algorithm. Appl Opt 2006; 45 5086-5091.

16 Shen XL, Kahn JM, Horowitz MA. Compensation for multimode fiber dispersion by adaptive optics. Opt Lett 2005; 30: 2985-2987.

17 Morales-Delgado EE, Farahi S, Papadopoulos IN, Psaltis D, Moser C. Delivery of focused short pulses through a multimode fiber. Opt Express 2015; 23: 9109-9120.

18 Katz O, Small E, Bromberg Y, Silberberg Y. Focusing and compression of ultrashort pulses through scattering media. Nat Photon 2011; 5: 372-377. (c) (1) (2) This work is licensed under a Creative Commons AttributionBY NC SA NonCommercial-ShareAlike 4.0 International License. The images or other third party material in this article are included in the article's Creative Commons license, unless indicated otherwise in the credit line; if the material is not included under the Creative Commons license, users will need to obtain permission from the license holder to reproduce the material. To view a copy of this license, visit http:// creativecommons.org/licenses/by-nc-sa/4.0/

(C) The Author(s) 2017

Supplementary Information for this article can be found on the Light: Science \& Applications' website (http://www.nature.com/lsa). 